\title{
Does the Surgical Timing and Decompression Alone or Fusion Surgery in Lumbar Stenosis Influence Outcome in Cauda Equina Syndrome?
}

\author{
Bharat Rajendraprasad Dave, Puspak Samal, Romin Sangvi, \\ Devanand Degulmadi, Denish Patel, Ajay Krishnan \\ Department of Spine Surgery, Stavya Spine Hospital and Research Institute, Ahmedabad, India
}

\begin{abstract}
Study Design: A retrospective comparative analysis of 64 patients with cauda equina syndrome (CES), who underwent either decompression alone (NF) or fusion (F) surgery.

Purpose: We compared the outcomes and timing effects.

Overview of Literature: CES can cause loss of autonomic control of vesicular function and lower limb neurological deficits. Prompt diagnosis and emergency surgery markedly improve outcome. Although decompression only is a mainstream technique, there is guarded recovery of vesicular dysfunction. Decompression ventrally in a narrow window requires manipulation of neural tissue in an already jeopardised critical canal and may accentuate irreversible damages. In F surgery, the adequate exposure leads to a lower neural manipulation.

Methods: Until January 2008, we treated CES with decompression (laminectomy and/or discectomy). However, from that month forward, all our single-level CES patients have received a fusion operation. In this study, characteristic categorical variables and outcomes were analysed.

Results: In a retrospective analysis of 64 patients, NF ( $n=37)$ and $F(n=27)$ who received treatment, we found that both groups improved significantly on follow-up in all objective parameters. Although, the comparison of clinical and functional outcome data between the two groups was statistically insignificant, the average value of objective outcome such as vesicular function, low back pain (LBP), and complications was better for patients in $\mathrm{F}$ group compared with NF group. However, the patient satisfaction for the $\mathrm{F}$ group was also lower, in view of their residual symptoms and disabilities. Contrary to common perceptions, we found that the timing of surgery does not influence the recovery rate for either approach.

Conclusions: Although both the techniques appear to be equally effective, the fusion approach overall showed a definite edge over non-fusion, with respect to reduced incidence of iatrogenic dural tears, LBP, and overall outcome, even despite the lower patient satisfaction.
\end{abstract}

Keywords: Polyradiculopathy; Cauda equina; Syndrome; Intervertebral disc displacement; Spinal fusion; Diskectomy; Lumbar stenosis

Received Jul 10, 2018; Revised Aug 19, 2018; Accepted Sep 4, 2018

Corresponding author: Ajay Krishnan

Department of Spine Surgery, Stavya Spine Hospital and Research Institute, near Nagari Hospital, Mithakhali, Ellisbridge, Ahmedabad, Gujarat, 380006, India

Tel: +91-9824302768, Fax: +91-79-26408174, E-mail: drajaykrishnan@gmail.com, drajaykrishnan@yahoo.co.in 


\section{Introduction}

Cauda equina syndrome (CES) is a rare consequence of lumbar disc herniation (LDH) [1]. It is even more uncommon in lumbar spinal stenosis (LSS) [1,2]. CES has been described as a symptom complex of resulting in low back pain (LBP), bilateral sciatica, saddle anesthesia, and variable motor weakness in the lower extremities, with bowel and bladder dysfunction [3]. CES has usually been considered the only absolute indication for surgical treatment in lumbar disorders. Although earlier studies of CES have reported poor prognosis, the overwhelming majority of modern reports have recommended that prompt diagnosis and emergency surgery result in markedly improved outcomes [3-5]. There are many literature reports confidently predicting outcomes only on the basis of presentation factors and timing of the surgery [3-5].

Laminectomy with or without discectomy is a common and widely accepted surgery for the management of LSS and LDH without CES $[4,6,7]$. Some authors have reported excellent early results with this procedure, although several have demonstrated that residual back pain, recurrent disc herniation, and disc degeneration and its consequences progress until later in life [8]. Still, it is questionable whether or not fusion addresses the potential adverse effects related to accelerated disc degenerative processes that are associated with discectomy in the years following surgery. There are recent reports suggesting improved functional outcome of decompression with fusion $[8,9]$.

When LDH alone is present with CES, there is usually a massive disc prolapse showing as a complete myelogram block. A decompression ventrally requires manipulation of neural tissue in an already jeopardized critical canal, which may accentuate irreversible damages. Fusion allows for the least neural manipulation, and with adequate exposure; however, no study has ever recommended fusion as a better alternative treatment option in LDH or LSS in CES cases, and a consensus is yet to be achieved.

This paper presents our retrospective analysis of the experience of treating CES at a single center. The primary aim of this investigation was to compare the clinical and functional outcomes between the non-fusion surgery (NF) and fusion surgery (F) CES treatment approaches. Our secondary goal was to determine if early surgery can yield a better prognosis.

\section{Materials and Methods}

This study was approved by the Institutional Ethical Committee of Stavy Spine Hospital \& Research Institute (SSHRI/CS/NS/CES/BRD/06/01-12) and informed consent was taken from all patients. From January 2003 to December 2011, a total of 1,864 and 1,684 patients received operations at Stavy Spine Hospital \& Research Institute, Ahmedabad, Gujarat, India for single level LDH and LSS, respectively. During this time, a total of 148 patients, with a variety of etiologies, also received operations for CES at our center. Only patients with CES due to single level LDH or LSS were reviewed and included in this study, and the minimum follow-up was set as 6 years. Data were collected from medical records and image databases. Patients who fulfilled the inclusion criteria were reviewed for demographics that included age, sex, body mass index, duration of LBP and leg pain, onset of bowel and bladder problems (acute or chronic), and also for neurologic issues (whether sensory and/or motor, whether unilateral or bilateral). Motor weakness was assessed using the Medical Research Council (MRC) grading scale of 0 to 5 . A power of root less than grade 3 was considered to be a significant weakness, and improvement of greater than grade 3 was considered to be a recovery. The severity of CES was categorized into two types. The first is called CESI (incomplete with impairment of bladder control), in which patients exhibited a spectrum of urinary difficulties but no urinary retention. The second, called CESR (retention with complete loss of bladder control), is where they had urinary retention. CES presentation was classified as acute or chronic. Chronic CES typically presents with a progressive LBP or radiculopathy and onset of vesicular dysfunction signaling the start of CES. The symptom duration of LBP and radiculopathy of more than 3 months was considered as chronic.

The radiological severity of LDH and LSS was analyzed using a qualitative grading system, using a grade from the Michigan State University (MSU) classification system [10] and morphological grades of stenosis according to Schizas et al. [11], respectively. Magnetic resonance imaging (MRI) scans were performed using 1.5 Tesla MRI scanners (Siemens, Erlangen, Germany). Magnetic resonance myelogram studies assessed the presence or absence of a complete block. The description of Schizas grading of stenosis is as follows (Fig. 1): grade A (nil or minor), grade B (moderate), grade C (severe), and grade D (extreme) [11]. 


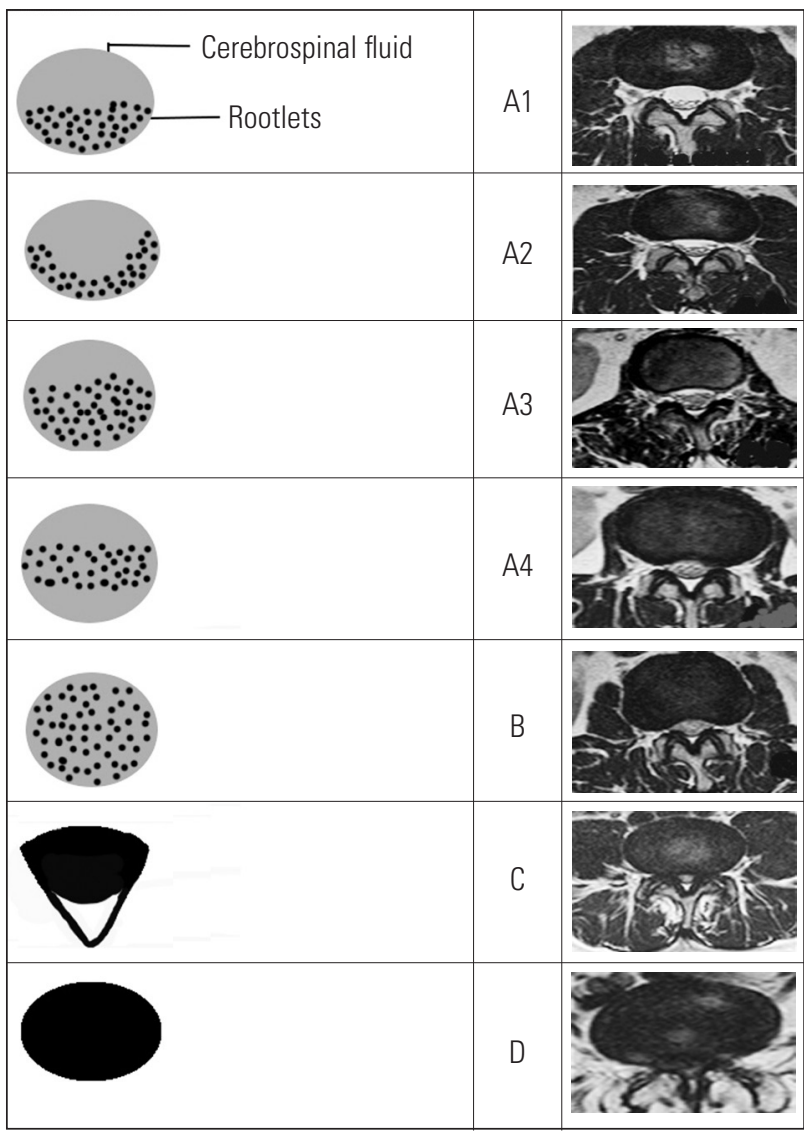

Fig. 1. Illustrative Schizas grades of lumbar spinal stenosis. Grades C and $\mathrm{D}$ are severe stenosis with least manipulation margin.
We evaluated for LDH (Fig. 2) using grades 1, 2, and 3 with type A, B, and C lesions [10]. Dynamic lateral radiographs were used to describe the presence of any instability.

All patients were operated under general anesthesia in a prone position. All the surgeries were performed by the senior authors (B.R.D., A.K.). Until January 2008, we treated CES with decompression (laminectomy and/ or discectomy). From 2008 onward, all patients were operated with trans-foraminal lumbar interbody fusion (TLIF). Pedicle screws with locally harvested bone grafts and an interbody cage were used in the TLIF procedure. Postoperatively, all patients were mobilized as their tolerance allowed and advised to undertake measured physiotherapy. The catheter was removed before discharge in the CESI group, but was left in place for the CESR patients. The catheter trial involved a change of catheters every 15 days for 3 months or recovery. All patients were reviewed regarding the time between onset and surgery, the reason for delay in surgery, type of surgery as between the F or NF alternatives, the time to the recovery of vesicular function (days), and the length of follow-up (months) as well as for complications.

The evaluation of neurologic and functional outcomes was done using the validated measures of the Oswestry

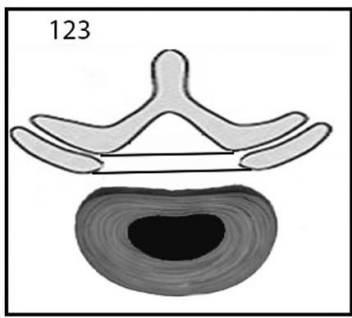

$1 C$

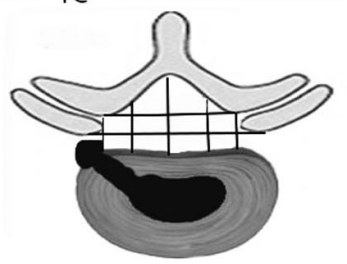

$2 C$

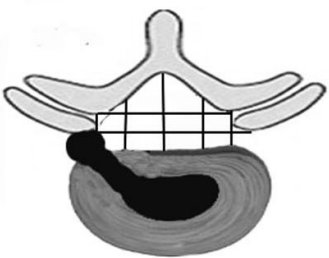

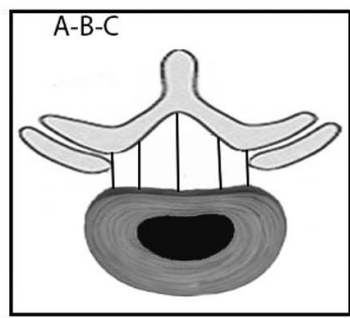

$2 A B$

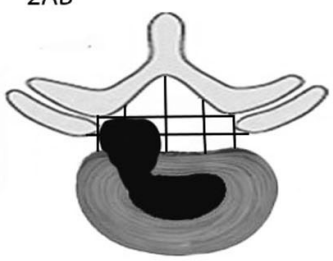

$3 A B$

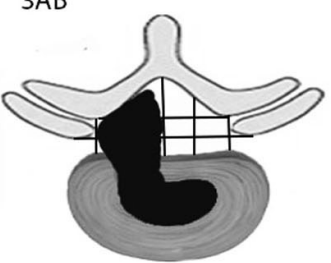

$1 \mathrm{~A}$

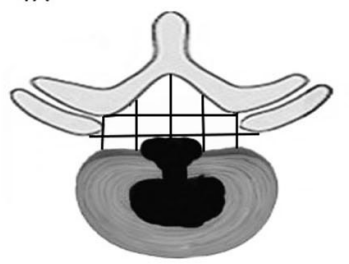

$2 \mathrm{~A}$

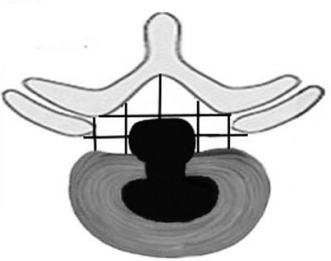

$3 \mathrm{~A}$

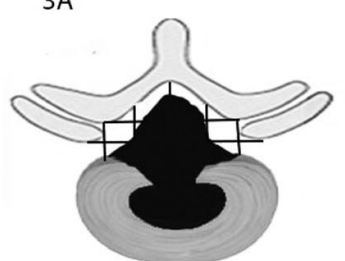

1B

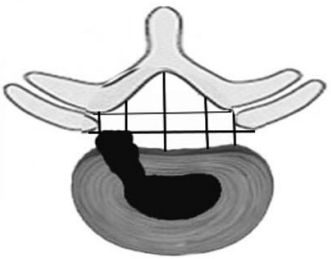

$2 B$

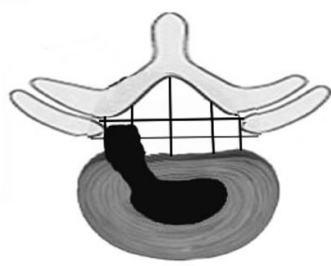

$3 B$

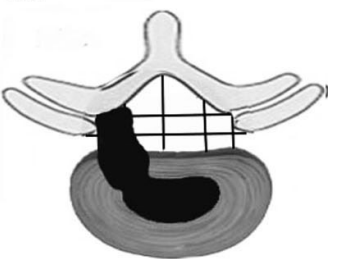

Fig. 2. Illustrative Michigan State University classification grades of lumbar disc herniation. Grade 3 indicates that more than $50 \%$ of the canal is compromised and that the dura with rootlets are dorsally shifted and stretched. 
Table 1. Comparison of demographic variables between the non-fusion and fusion groups

\begin{tabular}{|c|c|c|c|}
\hline Variable & Nonfusion ( $\mathrm{n}=37$ ) & Fusion ( $n=27)$ & $p$-value \\
\hline Age (yr) & $48.16 \pm 11.12(25-70)$ & $51.29 \pm 12.44(26-72)$ & \\
\hline \multicolumn{4}{|l|}{ Sex } \\
\hline Male & 25 & 22 & 0.62 \\
\hline Female & 12 & 5 & 0.33 \\
\hline Basal metabolic index & $23.61 \pm 4.03(17.5-34.3)$ & $24.56 \pm 4.36(19.4-34.6)$ & \\
\hline \multicolumn{4}{|l|}{ Primary case } \\
\hline LSS & 1 & 4 & 0.10 \\
\hline $\mathrm{LDH}$ & 36 & 23 & 0.71 \\
\hline Level of lesion & & & 0.6120 \\
\hline L5-S1 & 9 & 8 & \\
\hline L4-5 & 27 & 17 & \\
\hline L3-4 & 0 & 1 & \\
\hline $\mathrm{L} 2-3$ & 1 & 1 & \\
\hline LDH Michigan State University grade & & & 0.71 \\
\hline $3-A$ & 18 & 14 & \\
\hline $3-A B$ & 14 & 9 & \\
\hline $2-A$ & 3 & 1 & \\
\hline $2-A B$ & 1 & 2 & \\
\hline \multicolumn{4}{|l|}{ LSS Schizas grade } \\
\hline C & 5 & 3 & 0.80 \\
\hline $\mathrm{D}$ & 1 & 1 & 0.82 \\
\hline Time to surgery & $3.86 \pm 3.25(1-15)$ & $3.62 \pm 1.91(1-9)$ & 0.1845 \\
\hline$<48 \mathrm{hr}$ & 18 & 6 & \\
\hline$>48 \mathrm{hr}$ & 16 & 20 & \\
\hline$>7$ day & 7 & 3 & \\
\hline
\end{tabular}

Values are presented as mean \pm standard deviation (range) or number. LSS, lumbar spinal stenosis; LDH, lumbar disc herniation.

Disability Index (ODI) and Visual Analog Scale (VAS) score, which is a 10-point scale for LBP, leg pain, and leg tingling numbness. At follow-up, the quality of LBP was categorized into non-instability LBP (NILBP) and instability-related LBP (ILBP). If present, any constant backache not related they workload and not aggravated by loading or reloading, which felt more like a pulling or stiffness in the back, was labeled as NILBP. The pain in ILBP was typically associated with positional change, such as standing up from sitting, bending forward, and floor activities, or related to workloads that were aggravated by loading or reloading. This pain may have been constant or episodic. The urological outcome (recovered or not) was assessed. The final status was noted as recovered to normal, recovered to CESI status, or remained CESR.
Sexual dysfunction scoring on the basis of the Sexual Health Inventory in Males (SHIM) and a questionnaire to assess female sexual dysfunction was followed. The SHIM is a 5 -item questionnaire that is a validated screening tool for erectile dysfunction [12]. However, in conservative societies like India, a woman is expected to maintain silence when confronted with issues of her own sexuality. So instead of available validated female sexual dysfunction scores in the literature, we used a non-validated selfassessment questionnaire to assess female sexual dysfunction. The females were asked to give an overall score to categorize sexual function as good, fair, or poor. They were advised to consider factors of frequency, satisfaction, dryness, and pain to decide their score. A patient satisfaction index was used as a self-assessment tool to determine 
the overall satisfaction outcome [13]. The following recovery rates were assessed:

Vesicular recovery rate $=\frac{\text { No.of patients with complete vesicular recovery }}{\text { No.of patients with preoperative vesicular dysfunction }} \times 100$
Motor recovery rate $=\frac{\text { No.of patients with motor recovery }(\text { MRC }>\text { grade } 3)}{\text { No.of patients with preoperative motor weakness }(\text { MRC }<\text { grade } 3)} \times 100$

Complications were assessed and graded as major or minor. The former were those which had an effect on the final outcome, and the latter did not have such an impact.

Patient demographics and characteristic categorical variables were analyzed and the mean \pm standard deviation (minimum and maximum) for all applicable variables were calculated. Each category was compared by using appropriate statistical tools such as the Pearson correlations, unpaired Student $t$-test and paired $t$-tests. Statistical analysis was performed with IBM SPSS software ver. 20.0 (IBM Corp., Armonk, NY, USA). A $p$-value of $<0.05$ was considered to be statistically significant.

\section{Results}

Among a total of 64 patients (47 males and 17 females) with CES who were operated on over the time period defined in this study, 37 were in the NF group, with 25 males and 12 females. There were 27 patients in the F group, of which 22 were males and five were females. The follow-up period was of $86.21 \pm 7.47$ months (range, 74-101 months) for the NF group and 79.55 \pm 6.21 months (range, 72-95 months) in the F group. There were no statistically significant differences between the two groups with regard to any of these variables (Table 1). The following features were analyzed and summarized.

\section{Presentation}

Acute onset of symptoms was present in the LSS group for four patients (one NF and three F), but in the LDH group, 46 had this experience ( $29 \mathrm{NF}$ and $17 \mathrm{~F}$ ). Insidious chronic onset was present in one patient from the F group in LSS and 13 patients (seven NF and six F) with LDH lesions.

\section{Radiographic findings}

The most common level of lesions in our study was L4-5 in 45 patients (68.75\%), followed by L5-S1 in 17 patients (26.56\%). There was also one L3-4 case, and two had le- sions in the L2-3 region. In the NF group, the most common region was L4-5 $(n=27)$ followed by L5-S1 $(n=9)$ and L2-3 $(n=1)$. A total of 36 patients $(97.29 \%)$ had LDH, and they were categorized into four MSU grades: 3-A $(\mathrm{n}=18), 3-\mathrm{AB}(\mathrm{n}=14), 2-\mathrm{A}(\mathrm{n}=3)$, and $2-\mathrm{AB}(\mathrm{n}=1)$. One LSS patient with a Schizas grade of $\mathrm{D}$ was found in the NF group. In both the $2 \mathrm{~A}$ and $2-\mathrm{AB} \mathrm{LDH}$ cases, additional ligamentum flavum hypertrophy and disk space settling were present.

On the other hand, the most common level of lesion in the F group was L4-5, which was found in 17 cases (97.29\%), followed by L5-S1 in eight patients (26.5\%), and 1 each in L3-4 and L2-3. A total of 23 patients (85.18\%) had $\mathrm{LDH}$, and they were categorized into MSU grades of 3-A $(n=14)$ and 3-AB ( $n=9)$. Four of these F group patients had LSS, and of these, three had Schizas grade $\mathrm{C}$ and one was grade D. Again, there were no significant differences $(p=0.612)$ between groups with regard to any of these variables. All the LDH and LSS patients demonstrated complete myelographic block. One patient in the F group had instability.

\section{Onset to surgery delay}

A total of 24 cases (18 NF and six F, 37.5\%) received operations within 48 hours of onset, and in 36 cases (16 NF and 20 F, 56.2\%), this delay exceeded 48 hours. Ten patients had a delay more than 7 days, and of these, seven were in the NF group and three in the F group. In eight cases (six NF and two F), the delay was due to unawareness on the part of patients about the severity of their condition and they consequently neglecting it (all of these cases were CESI). In 26 patients (19 NF and seven F), delay was due to the primary care physicians in diagnosing CES, and in four cases (three NF and one F), delay occurred in obtaining a diagnostic study (MRI) or in seeking a specialist consultation. Two patients in the F group were delayed for 2 days because of their medically unfit status, and there was a planned delay in 24 patients. This category of delays was chosen by us to schedule the optimal next available morning operating room preparation for this group of patients. No emergency surgery was done since no patients presented in the evening or late night-time. Contrary to some expectations, we found no statistically significant differences in outcome with regard to surgical delay in our study, as distinguished by those who received operations before or after 48 hours from onset. 
Table 2. Comparison of the results between the non-fusion and fusion groups

\begin{tabular}{|c|c|c|c|}
\hline Variable & Nonfusion & Fusion & $p$-value \\
\hline \multicolumn{4}{|l|}{ LBP } \\
\hline Preoperative LBP & 13 & 14 & \\
\hline Non-instability LBP & 9 & 11 & \\
\hline Instability-related LBP & 6 & 0 & \\
\hline \multicolumn{4}{|l|}{ Leg pain } \\
\hline Preoperative present & 37 & 26 & 0.92 \\
\hline Preoperative absent & 0 & 1 & \\
\hline Residual leg pain & 5 & 1 & 0.22 \\
\hline \multicolumn{4}{|l|}{ Urologic dysfunction } \\
\hline Preoperative CESI & 12 & 7 & 0.67 \\
\hline Preoperative CESR ${ }^{\text {b) }}$ & 25 & 19 & \\
\hline \multicolumn{4}{|l|}{ Follow-up } \\
\hline Normal & 33 & 25 & \\
\hline CESI & 1 & 2 & \\
\hline CESR & 3 & 0 & \\
\hline Vesicular recovery rate $(\%)$ & 89.18 & 92.59 & \\
\hline Motor recovery rate (\%) & 43.75 & 46.67 & \\
\hline
\end{tabular}

LBP, low back pain; CES, cauda equina syndrome.

a) Incomplete with impairment of bladder control. ${ }^{b}$ Retention with complete loss of bladder control.

\section{Low back pain}

A total of 27 patients had LBP at presentation (14 F and $13 \mathrm{NF}$ ). The preoperative VAS score was $2.13 \pm 2.98$ (range, $0-7$ ) in the NF group, which improved to $2.02 \pm 1.87$ (range, $0-6)$, whereas the preoperative VAS score in the F group was $2.85 \pm 3.18$ (range, $0-9$ ), and it improved to $0.84 \pm 1.14$ (range, 0-6). All these patients improved significantly $(p<0.05)$ at follow-up. After surgery, the NILBP at followup was present in both the NF $(n=11)$ and the $F(n=9)$ groups and six had ILBP in the NF group but none in the F group. However this difference was statistically insignificant (Table 2).

\section{Leg pain}

Except for one in the F group, all patients (98.4\%) had leg pain at presentation. The preoperative VAS score in the NF group was $6.48 \pm 1.01$ (range, 5-8) and improved to $0.37 \pm 0.89$ (rang, $0-3$ ) at final follow-up. The preoperative VAS score in the F group was $6.33 \pm 1.73$ (range, $0-8$ ) and improved at final follow-up to $0.11 \pm 0.57$ (range, $0-3$ ). The

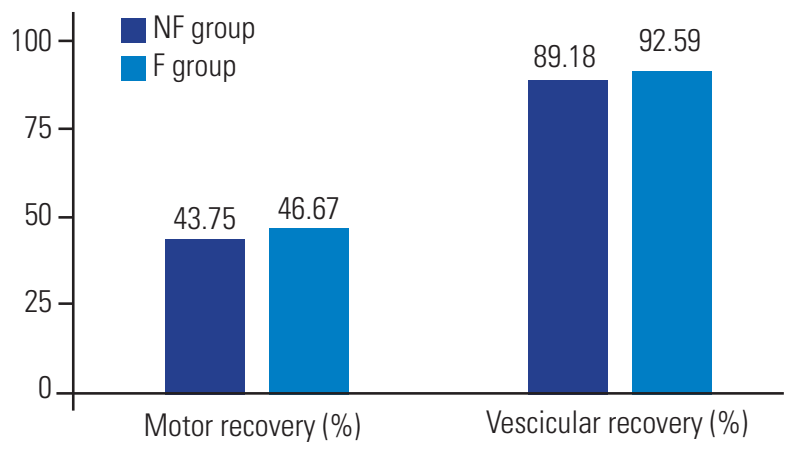

Fig. 3. Comparisons of motor and vesicular recovery scores between the NF and F groups. NF, non-fusion surgery; F, fusion surgery.

improvement in both groups was significant compared with the preoperative VAS score; however, residual leg pain was present in one patient $(3.8 \%)$ in the $\mathrm{F}$ and five $(13.5 \%)$ in the NF group, with a VAS score $2.4 \pm 0.54$ (range, $2-3)$. There was no statistically significant difference $(p=0.22)$ between the two groups (Table 2 ).

\section{Urologic dysfunction}

At presentation, 12 patients in the NF and seven in the F group had CESI, whereas 25 patients in the NF and 19 in the F group had CESR. At follow-up, all CESI patients in both the NF and F groups had complete recovery. In the NF group among all 25 CESR patients, 19 improved completely, although one improved to CESI with residual hesitancy, and three patients did not have improved retention. Among the 19 CESR patients in the F group, all but two improved to normal, although both had improved to CESI with residual urgency/hesitancy. The differential recovery was statistically insignificant in both the F and NF groups though the average recovery was greater in the $\mathrm{F}$ group (Table 2).

The vesicular recovery rate was $91.66 \%$ in patients operated on within 48 hours and was $90 \%$ in those whose operations occurred after 48 hours. The vesicular recovery rate was $89.18 \%$ in NF patients and $92.59 \%$ in the F group (Fig. 3). These differences were not statistically significant. Constipation was present in 26 patients of the NF group compared with only 14 in the F group at presentation. At final follow-up, seven patients in the NF group and four in the F group had residual constipation. However, these outcomes were also not statistically significant. 


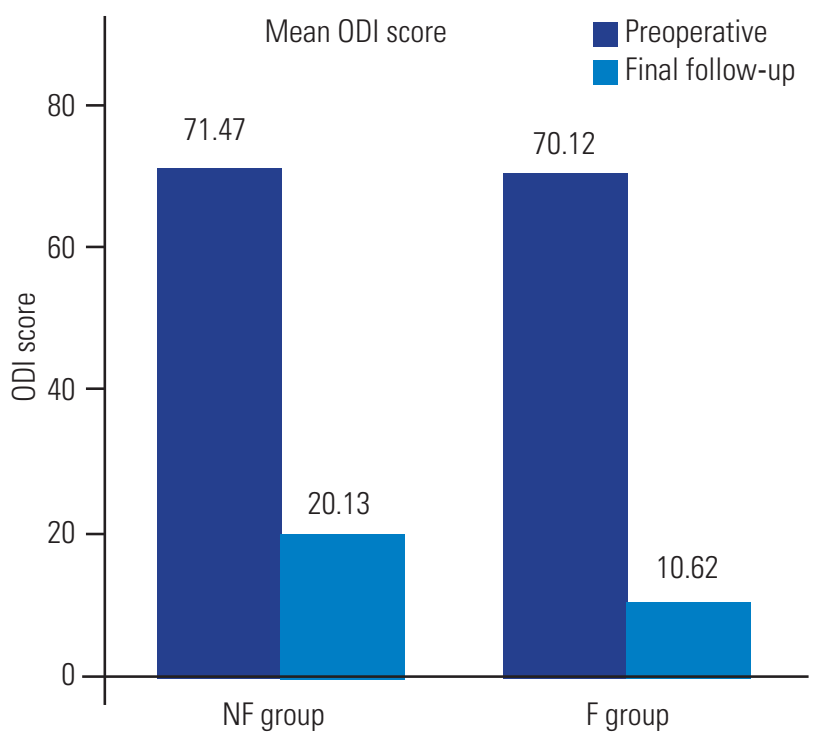

Fig. 4. Comparison of ODI scores between the NF and F groups. ODI, Oswestry Disability Index; NF, non-fusion surgery; F, fusion surgery.

\section{Motor and sensory dysfunction}

Preoperative motor weakness in the lower limbs was present in 31 patients (16 NF and $15 \mathrm{~F}$ ) and five (two NF and three F) of them had bilateral weakness. At follow-up, 15 patients (seven NF and eight F) had improvement in their power. The motor recovery rate was $50 \%$ in patients who received operations within 48 hours and $45.83 \%$ for those occurring after 48 hours. The motor recovery rate in the NF group was $43.75 \%$, and for the F group, it was $46.67 \%$ (Fig. 3), although again these results were not statistically significant.

There was variable tingling numbness present in all except one patient in both the NF and F groups at presentation. At final follow-up, this was present in 49 patients (31 NF and $18 \mathrm{~F}$ ). No significant differences in motor recovery and tingling numbness were found in either the $\mathrm{F}$ or the NF group.

\section{Functional improvement}

The overall average preoperative ODI was $70.9 \pm 13.5$ (range, 44.4-100) and at follow-up was down to $16.5 \pm 20.5$ (range, 0-71.10). This improvement was statistically significant and more than the minimal level of significant clinical difference. The average preoperative ODI scores of the NF group were $71.47 \pm 13.33$ (range, 44.4-100) and in the F group was $70.12 \pm 14.09$ (range, 48.8-97.7). The
Table 3. Comparison of the complications between the non-fusion and fusion groups

\begin{tabular}{lccc} 
Complications & Nonfusion & Fusion & $p$-value \\
Urinary tract infection & $11(29.72)$ & $6(22.22)$ & 0.60 \\
Dural tear & $10(27.00)$ & $2(3.12)$ & 0.09 \\
Superficial infection & 1 & 2 & 0.40 \\
\hline Values are presented as number (\%). & &
\end{tabular}

follow-up ODI was 20.13 \pm 20.42 (range, 0-66.67) in the NF group and 10.62 \pm 18.85 (range, $0-71.1$ ) in the F group (Fig. 4). This difference in ODI in the two groups was insignificant statistically although the average improvement was greater in the $\mathrm{F}$ group. The patient satisfaction index was $2.35 \pm 0.78$ (range, $1-4$ ) in the NF and $1.77 \pm 0.64$ (range, $1-3$ ) in the F group, but it was statistically insignificant.

\section{Sexual dysfunction}

At follow-up, according to the SHIM scale, six patients (four NF and two F, 12.76\%) had severe erectile dysfunction. Out of 32 CESR male patients, 10 (seven NF and three F, 31.25\%) had moderate to severe erectile dysfunction. Of the remaining 15 CESI male patients, six (four NF and two F, 40\%) had moderate to severe erectile dysfunction. Female sexual function was poor in eight (five NF and three F), fair in seven (six NF and one F) and rated as good in only two (one NF and one F). There was no statistically significant difference between the CESI/CESR or NF/F groups.

\section{Complications}

Out of 64 patients, 17 (26.56\%, 11 NF and six F) had urinary tract infections and all responded to medical treatment. Dural tear occurred in two patients $(3.12 \%)$ in the $\mathrm{F}$ group and 10 (27\%) in the NF group; however, this difference was statistically insignificant $(p=0.09)$. Drain removal was delayed in such cases, and stitches were utilized at the drain site on removal. One patient had developed a superficial infection but was managed conservatively and responded well (Table 3 ).

\section{Discussion}

The spinal cord terminates as the conus medullaris at the lower level of the L1 vertebrae and continues as a bundle 


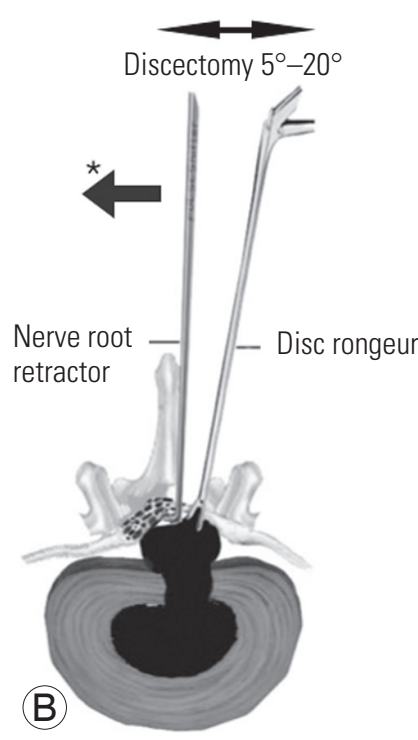

(A)

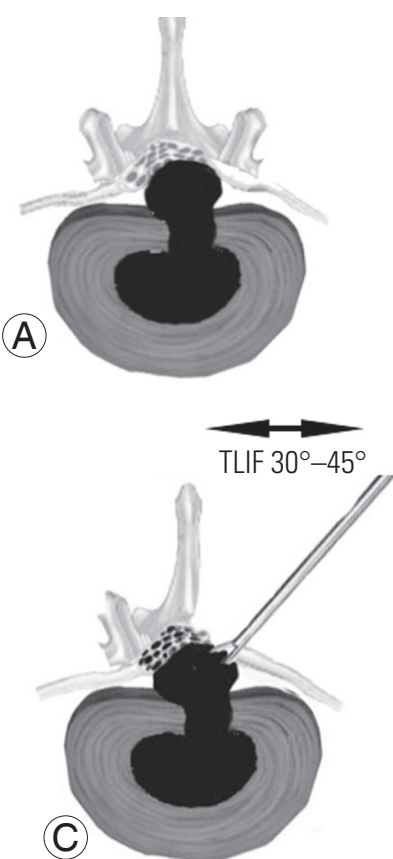

Fig. 5. Illustration showing a massive discogenic stenosis (A) if a discectomy is done; (B) it needs a dura retractor that pushes ( ${ }^{*}$ arrow), the compromised stretched dura, and neural tissue medially to make space for a disc rongeur to grab the fragment; (C) in case of a TLIF, the space created by facetectomy gives better angulation of instruments and requires the least manipulation. TLIF, trans-foraminal lumbar interbody fusion.

of nerve roots called the cauda equina [14]. CES is a not so uncommon neurological disorder resulting from lesions of the nerves of cauda equina. Its incidence is reported to be approximately $2 \%$ of all operated LDH cases [1]. The incidence in our series was $3.16 \%$ and $0.29 \%$ in LDH and LSS cases, respectively. The pathophysiology of pain and deficit in LDH is understood to be due to compression, chemical irritation, vascular congestion, neuroischemia, and autoimmune response in cauda equina $[3,15]$.

Risk factors associated with LDH include obesity, male gender, age $>40$ years, heavier loading during occupational and leisure time activities, and a history of back disorders [16]. Men in their fourth and fifth decades of life are prone to disc herniation and subsequent progression to CES [17]. In this study, 47 patients (73.43\%) were males with $59.37 \%$ in their 40 s and 50 s, with the concomitant propensities toward CES. Six males were laborers performing heavy-duty activities. Approximately $70 \%$ of the patients have a history of disc height loss by chronic LBP, with CES developing as the final episode, although $30 \%$ of the patients in this study presented with CES as the first manifestation of the LDH [4].
In our sample, $53 \mathrm{LDH}$ patients (82.81\%) had acute onset as compared with six (9.37\%) who became acute after chronic onset symptoms, and five patients had LSS (0.29\%). A clinical disease state almost always manifests as a spectrum of disorders and CES is no exception. In our study, only one case proceeded directly to CES without LBP or radiculopathy but all other patients had typical manifestations. The most common level to be affected in CES is L4-5 followed by L5-S1 [1]. This was similar in our study with L4-5 level in $68.75 \%$ of the cases, followed by L5-S1 in $26.56 \%$ and only rare cases in the L2-3, L3-4 levels.

Various classification systems of CES have previously been reported. These include categories based on the acute or chronic nature of the LBP and lower limb pain, or based on electrophysiology $[3,18]$. Although the classifications mentioned are useful for documentation, for prognosticating outcome, and also for medico-legal purposes, nonetheless, CES has been best classified into two divisions: CESI and CESR [5].

MRI is the investigation method of choice for CES patients. Computed tomography myelography is equally useful in cases where MRI is contraindicated. The Schizas morphologic classification for LSS and the MSU system for LDH allow good clinical implementation for the severity of radiological stenosis. In all five of the LSS patients, a severe to extreme grade (grade D) of stenosis was present. Similarly, in LDH, 54.24\% ( $\mathrm{n}=32$ ) had 3-A followed by $38.98 \%(\mathrm{n}=23) 3-\mathrm{AB}$ grade lesions. These have a greater degree of LDH severity and are more likely to cause CES [10]. All the patients in our study had a complete myelogram block, signifying a severe degree of compression with massive disc prolapse $[19,20]$.

A major controversy in this field is whether the time to surgery from onset influences the final outcome. Some studies support the view that early surgery does influence recovery $[4,7]$ but others do not $[17,20]$. In our investigation, there was no significant correlation between onset to surgery delay and final outcome. Ten patients (five CESR and five CESI) who were operated on after a considerable delay of more than 7 days recovered well with a mean ODI score of $17.11 \pm 20.06$ (range, $0-67$ ) and vesicular recovery rates of $90 \%$. This is in contrast to the few earlier studies reporting a poor outcome after 7 days [21]. The timing between the onset of CES and surgery varied from 1 to 15 days, with average of 4 days. The usual causes for delay in the literature have been failure to diagnose CES by 
primary physicians, unavailability of adequate medical facilities to investigate, and delay in surgery until optimum operative circumstances were available $[7,20]$. Although all the CESR patients presented earlier, in eight CESI cases there was a significant delay due to patient's attitude and neglect in coming forward. Primary physicians delayed in referring 28 patients for specialist consultation because of delayed MRI acquisition and unawareness of the emergency situation. Twenty-four patients after presentation to us were further delayed for the next earliest operative day list. But all these circumstances did not affect the outcome statistically.

The cauda equina conveys sensory information from both the sacral dermatomes, which are motor nerve fibers innervating the lower sacral myotome skeletal muscles, and the sacral parasympathetic fibers. Thus, in patients with cauda equina lesions, the finding of pronounced sexual dysfunction, perineal sensory deficit, and electromyography abnormalities is not unexpected. Sexual dysfunction can cause extremely distressing effects on self-esteem and relationships. Following surgery, sexual dysfunction persists in a significant number of CES patients, and in addition, increasing age seems to influence this situation [22]. There was no significant difference in sexual functional outcomes in the F compared with the NF groups in our data.

Although earlier studies have reported decompression as a routine treatment modality in LDH and LSS, there is a paucity of evidence regarding the fusion approach. There are no reports addressing the recommendation of fusion in CES either, although a wider use of decompression has been routinely advised $[3,7,17,23]$. Recurrent $\mathrm{LDH}$ is a common cause of poor outcomes after LDH surgery, with reported incidences of reoperation from 5\% to $24 \%[8,9,23]$. LDH may lead to progressive degenerative disc disease (DDD) and a late segmental instability, with chronic LBP frequently necessitating reoperation [19]. Studies have reported high satisfaction rates in terms of LBP and sciatica after fusion as compared with decompression alone $[8,9]$. Although decompression has been routinely chosen as a standard treatment regimen in LSS, lately fusion is being more frequently advised, especially in the presence of subtle predisposing factors of instability (facet orientation and disc height loss $>30 \%$ ) [24]. CES does require more extensive decompression with laminectomy $[3,7,23]$ to avoid further neurological worsening due to manipulative neural injury in an already severely compromised canal (Fig. 5) [17,20]. Excessive manipulation during discectomy in a severely compromised canal has been reported as a cause of further deficit [23]. Therefore, a minimally invasive approach to this type of $\mathrm{LDH}$ is also not recommended. For the same reason, theoretically lesser manipulative approaches like ventral endoscopic transforaminal decompression may yield better results, but these need to be studied further before they might be recommended in the future. In our study, dural tear occurred in two patients in the F group as compared with 10 from the NF group, though this difference was not statistically significant. Dural tear is a complication of iatrogenic injury, and the larger numbers from the NF group reflect the more extensive manipulations that were done during decompression in severely compromised canals and therefore encountering the larger likelihood of inadvertent errors (Fig. 5). The number of urinary tract infections, though statistically insignificant, was greater in the NF group ( $\mathrm{n}=11,29.72 \%)$ compared with the F group $(\mathrm{n}=6$, $22.22 \%)$. This indirectly relates to the longer duration of catheterization needed in the NF group, thus increasing the risk of infections and the need for the urologist to manage them.

Wide decompression leads to a greater incidence of postoperative instability, progressive stenosis, and recurrence of LBP or leg symptoms [25-27]. Biomechanical studies have also reported a positive correlation between the extent of decompression and instability [24]. This may be due to an iatrogenic pars fracture or an overly aggressive resection of posterior elements leading to accelerated DDD [28].

The ODI and the VAS scores for LBP and leg pain improvement were significant in all the patients. Although statistically insignificant, the average score improvements regarding ODI, VAS (LBP and leg pain), and vesicular and motor recovery were more in favor of the $\mathrm{F}$ group (Figs. 3, 4). The ILBP in the NF group is suggestive of pain on change of position leading to temporary mechanical derangement at the affected segment. Moreover, in the NILBP patients, the ODI at follow-up of the NF group was significantly higher and therefore categorized as moderated disability, whereas the F group NILBP patients were considered to have minimal disability. The onset of new LBP was more common in the NF group. The cases of NILBP were present in nine F group and 11 NF group patients, but this was not disabling, though troublesome. Fusion added to stiffness and backaches more often in the 
$F$ than in the NF group.

The patient satisfaction index values in our study for the $\mathrm{F}$ and NF groups were $1.77 \pm 0.64$ (range, 1-3) and $2.35 \pm 0.78$ (range, $1-4$ ), respectively. These were much lower than typical good objective functional, neurological, and urological outcomes. The suboptimal patient satisfaction index values provided by our patients seem to be due to the persistence of lower limb tingling, numbness in $76.56 \%(n=49)$, variable residual subjective disability, and sexual dysfunction. This type of outcome can lead to a high risk of patients seeking multiple specialist consultations, which might in turn produce competitive and provocative opinions, compounding the risk of litigation. As an alternative, it has been recommended that patients should accept their residual disability and seek the best social and medical rehabilitation. In any case, we have been singularly lucky to not have faced litigation in spite of the delays in surgery and the residual consequences in outcomes.

Our study has several inadequacies, especially the ones usually characteristic of retrospective analyses. Of course, studies with matched controls are necessary before we can determine the full clinical value of fusion in CES. In our investigation lesser affected patients may have been in the F group, although the demographic and presentation data of our subjects do not suggest this. The disadvantages of fusion are its greater cost, longer hospital stay, new problems of adjacent segment disease, and instrumentationrelated complications [29]. These were not evaluated. It is also unknown whether the more recent alterations in treatment have resulted in better outcomes or if these contemporary results are largely due to the now greater surgical experience of the surgeons. Sexual dysfunction scores were not documented preoperatively, and only follow-up scores were available. Female sexual dysfunction assessment was done by a non-validated outcome measure, because the use of scores to define female sexual dysfunction is confounded by complex cultural expectations and taboos of various societies. In a conservative society like India, a woman is expected to maintain silence when confronted with issues of her own sexuality [30]. Therefore, a self-made modified questionnaire was used. The patient feedback we obtained regarding vesicular dysfunction was purely subjective, and no supportive urodynamic studies to document and compare them were done, and as result, it cannot be judged whether they are due to compensations developed by the body or true vesicular recovery.
However, urodynamic studies can show a serious disturbance of bladder function after cauda equina compression without the patient having any complaints [28]. A new prospective study is underway at our center, which should generate better recommendations in the near future regarding this issue.

It should be explicitly recognized that at present CES has a proportionately high medico-legal profile. Although our study does not definitively show that early surgery is always best, the ability to scientifically reach such a conclusion might remain elusive. Although our results are very encouraging regarding delayed surgery, it is presently a logical rather than an evidence-based recommendation to do the surgery at the earliest possible time.

\section{Conclusions}

Although both techniques appear to be equally effective, our study supports the view that fusion surgery provides better average outcomes than non-fusion surgery for CES on the basis of objective parameters and with fewer complications. Both techniques may result in some residual consequences, which can leave the patients considering that the results were not entirely satisfactory. This subjective disappointment is largely the reason for a variety of medico-legal issues. A proper multicenter prospective trial needs to be done before recommending guidelines for both the timing and type of surgery that might contradict the results we have found regarding the timing and type of surgery in our study.

\section{Conflict of Interest}

No potential conflict of interest relevant to this article was reported.

\section{References}

1. Gardner A, Gardner E, Morley T. Cauda equina syndrome: a review of the current clinical and medicolegal position. Eur Spine J 2011;20:690-7.

2. Johnsson KE, Sass M. Cauda equina syndrome in lumbar spinal stenosis: case report and incidence in Jutland, Denmark. J Spinal Disord Tech 2004;17:3345.

3. Gitelman A, Hishmeh S, Morelli BN, et al. Cauda equina syndrome: a comprehensive review. Am J Or- 
thop (Belle Mead NJ) 2008;37:556-62.

4. Shapiro S. Medical realities of cauda equina syndrome secondary to lumbar disc herniation. Spine (Phila Pa 1976) 2000;25:348-51.

5. Gleave JR, Macfarlane R. Cauda equina syndrome: what is the relationship between timing of surgery and outcome? Br J Neurosurg 2002;16:325-8.

6. Kostuik JP, Harrington I, Alexander D, Rand W, Evans D. Cauda equina syndrome and lumbar disc herniation. J Bone Joint Surg Am 1986;68:386-91.

7. Ahn UM, Ahn NU, Buchowski JM, Garrett ES, Sieber AN, Kostuik JP. Cauda equina syndrome secondary to lumbar disc herniation: a meta-analysis of surgical outcomes. Spine (Phila Pa 1976) 2000;25:1515-22.

8. LaMont RL, Morawa LG, Pederson HE. Comparison of disk excision and combined disk excision and spinal fusion for lumbar disk ruptures. Clin Orthop Relat Res 1976;(121):212-6.

9. Vaughan PA, Malcolm BW, Maistrelli GL. Results of L4-L5 disc excision alone versus disc excision and fusion. Spine (Phila Pa 1976) 1988;13:690-5.

10. Mysliwiec LW, Cholewicki J, Winkelpleck MD, Eis GP. MSU classification for herniated lumbar discs on MRI: toward developing objective criteria for surgical selection. Eur Spine J 2010;19:1087-93.

11. Schizas C, Theumann N, Burn A, et al. Qualitative grading of severity of lumbar spinal stenosis based on the morphology of the dural sac on magnetic resonance images. Spine (Phila Pa 1976) 2010;35:191924.

12. Cappelleri JC, Rosen RC. The Sexual Health Inventory for Men (SHIM): a 5-year review of research and clinical experience. Int J Impot Res 2005;17:307-19.

13. Toyone T, Tanaka T, Kato D, Kaneyama R, Otsuka M. Patients' expectations and satisfaction in lumbar spine surgery. Spine (Phila Pa 1976) 2005;30:2689-94.

14. Arce D, Sass P, Abul-Khoudoud H. Recognizing spinal cord emergencies. Am Fam Physician 2001;64:631-8.

15. Sekiguchi M, Kikuchi S, Myers RR. Experimental spinal stenosis: relationship between degree of cauda equina compression, neuropathology, and pain. Spine (Phila Pa 1976) 2004;29:1105-11.

16. Kostova V, Koleva M. Back disorders (low back pain, cervicobrachial and lumbosacral radicular syndromes) and some related risk factors. J Neurol Sci
2001;192:17-25.

17. Shapiro S. Cauda equina syndrome secondary to lumbar disc herniation. Neurosurgery 1993;32:743-6.

18. Shi J, Jia L, Yuan W, et al. Clinical classification of cauda equina syndrome for proper treatment. Acta Orthop 2010;81:391-5.

19. Satoh I, Yonenobu K, Hosono N, Ohwada T, Fuji T, Yoshikawa $\mathrm{H}$. Indication of posterior lumbar interbody fusion for lumbar disc herniation. J Spinal Disord Tech 2006;19:104-8.

20. Kostuik JP. Medicolegal consequences of cauda equina syndrome: an overview. Neurosurg Focus 2004;16:e8.

21. Busse JW, Bhandari M, Schnittker JB, Reddy K, Dunlop RB. Delayed presentation of cauda equina syndrome secondary to lumbar disc herniation: functional outcomes and health-related quality of life. CJEM 2001;3:285-91.

22. Podnar S, Oblak C, Vodusek DB. Sexual function in men with cauda equina lesions: a clinical and electromyographic study. J Neurol Neurosurg Psychiatry 2002;73:715-20.

23. McLaren AC, Bailey SI. Cauda equina syndrome: a complication of lumbar discectomy. Clin Orthop Relat Res 1986;(204):143-9.

24. Abumi K, Panjabi MM, Kramer KM, Duranceau J, Oxland T, Crisco JJ. Biomechanical evaluation of lumbar spinal stability after graded facetectomies. Spine (Phila Pa 1976) 1990;15:1142-7.

25. Vik A, Zwart JA, Hulleberg G, Nygaard OP. Eight year outcome after surgery for lumbar disc herniation: a comparison of reoperated and not reoperated patients. Acta Neurochir (Wien) 2001;143:607-10.

26. Jonsson B, Annertz M, Sjoberg C, Stromqvist B. A prospective and consecutive study of surgically treated lumbar spinal stenosis: part II: five-year follow-up by an independent observer. Spine (Phila Pa 1976) 1997;22:2938-44.

27. Xia Y, Ishii K, Matsumoto M, Nakamura M, Toyama Y, Chiba K. Radiographic predictors of residual low back pain after laminectomy for lumbar spinal canal stenosis: minimum 5-year follow-up. J Spinal Disord Tech 2008;21:153-8.

28. Zander T, Rohlmann A, Klockner C, Bergmann G. Influence of graded facetectomy and laminectomy on spinal biomechanics. Eur Spine J 2003;12:427-34.

29. Hijji FY, Narain AS, Haws BE, et al. Does day of 
surgery affect hospital length of stay and charges following minimally invasive transforaminal lumbar interbody fusion? Clin Spine Surg 2018;31:E291-5.
30. Singh JC, Tharyan P, Kekre NS, Singh G, Gopalakrishnan G. Prevalence and risk factors for female sexual dysfunction in women attending a medical clinic in south India. J Postgrad Med 2009;55:113-20. 